\title{
Measurement of the Mie-Grüneisen equation of state for polyimide
}

\author{
CHEN Hua $^{1 *}$, TANG WenHui $^{1}$, RAN XianWen $^{1}$, ZHANG MingJian $^{2} \&$ XU ZhiHong ${ }^{1}$ \\ ${ }^{1}$ Institute of Engineering Physics, College of Science, National University of Defense Technology, Changsha 410073, China; \\ ${ }^{2}$ School of Physics and Science Technology, Southwest Jiaotong University, Chengdu 610031, China
}

Received June 12, 2012; accepted November 5, 2012; published online December 6, 2012

\begin{abstract}
An equation of state (EOS) is a thermodynamic equation describing the state of matter under a given set of physical conditions, and the Mie-Grüneisen EOS is a widely used EOS for solid materials. Polyimide is a typical complex high-molecular polymer of imide monomers which is widely used in the manufacture of parts for aerospace technology and for fabricating the inertial fusion energy shells (IFE). When a spacecraft collides with a meteoroids (or orbital debris) or the IFE shells are radiated by high power laser, the polyimide used in the equipment is in high pressure state. In order to study the safety of the spacecraft and IFE shells, the EOS of polyimide should be determined. In our research, the three key parameters of the Mie-Grüneisen EOS based on the shock adiabat for polyimide have been determined with two-stage light-gas gun experiments, i.e. the bulk speed of sound of polyimide is determined as $2.62 \pm 0.22 \mathrm{~km} / \mathrm{s}$, the linear Hugoniot slope coefficient is determined as $1.25 \pm 0.063$, and the Grüneisen parameter at initial state is determined as $1.53 \pm 0.135$. The Mie-Grüneisen EOS for polyimide is finally obtained, and the parameter values are proved reliable via the comparison of Grüneisen parameter value calculated from two different theoretical models using the experimental data.
\end{abstract}

polyimide, Hugoniot parameters, the Grüneisen parameter, two-stage gas gun

Citation: Chen H, Tang W H, Ran X W, et al. Measurement of the Mie-Grüneisen equation of state for polyimide. Chin Sci Bull, 2013, 58: 585-588, doi: 10.1007/ s11434-012-5602-4

An equation of state (EOS) is a thermodynamic equation describing the state of matter under a given set of physical conditions. Equations of state are useful in describing the properties of gas, fluids, mixtures of fluids, solids, and even the interior of stars. The Mie-Grüneisen EOS [1] which has many variations is a widely used EOS for solids, and one of the variations is the Mie-Grüneisen EOS based on the shock adiabat:

$$
p=p_{\mathrm{H}}+\Gamma \rho\left(e-e_{\mathrm{H}}\right),
$$

where

$$
\begin{gathered}
p_{\mathrm{H}}=c_{0}^{2}\left(v_{0}-v\right) /\left[v_{0}-s\left(v_{0}-v\right)\right]^{2}, \\
e_{\mathrm{H}}=\frac{1}{2} p_{\mathrm{H}}\left(v_{0}-v\right),
\end{gathered}
$$

\footnotetext{
*Corresponding author (email: chenhua1020@nudt.edu.cn)
}

where $p$ is pressure, $e$ is specific energy, $v$ is specific volume, $c_{0}$ and $s$ are the Hugoniot parameters in the linear relationship between the shock wave velocity $D$ and the particle velocity $u\left(D=c_{0}+s u\right)$, in general, $c_{0}$ is the bulk speed of sound, $s$ is linear Hugoniot slope coefficient and $\Gamma$ is the Grüneisen parameter.

The polyimide (PI) is a typical complex high-molecular polymer of imide monomers. It is well-known for excellent properties, such as being light weight, having thermal stability, good chemical resistance, low electrical conductivity, large radiation resistance, high tensile strength, large elastic module, and so on. Due to the excellent properties, polyimides are widely used along with composites based on polyimide fibers in the manufacture of parts for aerospace technology [2,3], polyimide films located in the surface layer are used to protect the spacecraft's electronic equipment from damage by low temperature in the space, and polyimide resin are used to produce the solar cell arrays. Polyimides are also used for fabricating the inertial fusion 
energy (IFE) shells [4,5] and the high-quality millimeter-sized polyimide shells have been fabricated by vapor deposition polymerization and by removing nonvolatile solvent.

Many spacecrafts work in the low earth orbit (LEO). There are a lot of meteoroids and orbital debris (MOD). Additionally, there are many kinds of aerospace rays. Polyimides used in the spacecraft surface layer would be in high-pressure state when the spacecraft collides with a meteoroid or orbital debris with hypervelocity, or is radiated by the aerospace rays [6-11]. Polyimides used in the IFE shells are also in the high-pressure state when radiated by powerful laser. In order to improve the safety of spacecraft and IFE facility, it is necessary to study the EOS of polyimide to describe its state.

The two-stage light-gas gun [12] is an effective tool to measure the EOS of materials, and our measurement experiments were conducted in the gas-gun system laboratory of Southwest Jiaotong University with $24 \mathrm{~mm}$ diameter two-stage light-gas gun at impact velocities between 2.0 and $5.0 \mathrm{~km} / \mathrm{s}$. The polyimide used in the experiment is made in Shanghai Research Institute of Synthetic Resins, which is polymerized with 4,4'-oxygen support dual-phthalic anhydride (single ether-anhydride) and 4,4'-oxydianiline.

The schematic of the experiment system is shown in Figure 1 . The projectile consists of a flyer holder and a flyer. These flyers are $2-3 \mathrm{~mm}$ thickness and $23 \mathrm{~mm}$ normal diameter. They are composed, respectively, of aluminum, copper and tantalum. The sample has two parts, the first part is $30 \mathrm{~mm}$ in diameter and $2-3 \mathrm{~mm}$ thick, the second part is $12 \mathrm{~mm}$ in diameter and 2-3 mm thick. A shockwave is generated and propagate through the sample after which is impacted by the flyer. Across a shockwave there is always an extremely rapid rise in pressure, temperature and density of the medium. The quartz fiber-optic pins which are located closely to the back surface of the polyimide, are sensitive to the pressure, and are used to translate the pressure signals to light signals [13]. The pyrometer translates the light signals to electrical signals which are finally recorded by an oscillograph.

The magnet velocity induced system is used to measure the velocity of flyer, and the accuracy is about $0.1 \%$. Figure
2 is a typical oscilloscope record obtained from quartz fiber-optic pins in the experiments. It can be found that the jump signals of the pins are very clear, therefore we can accurately obtain the travel time $\Delta t$ between the time given by taking an average from pin 1 and 2 and the shock wave reaching pin 3. As the thickness $d$ of the second part is measured beforehand, the shock wave velocity $D$ can be obtained as

$$
D=d / \Delta t
$$

With the flyer velocity $W$ and the shock wave velocity $D$, the particle velocity $u$ and the shock wave pressure of the target can be calculated by the shock wave equations:

$$
\begin{gathered}
u=\frac{-b-\sqrt{b^{2}-4 a h}}{2 a}, \\
p=\rho_{0 \mathrm{t}} D u,
\end{gathered}
$$

where $a=\rho_{0 \mathrm{f}} s_{\mathrm{f}}, b=-\left(\rho_{0 \mathrm{t}} D+\rho_{0 \mathrm{f}} c_{0 \mathrm{f}}+2 \rho_{0 \mathrm{f}} s_{\mathrm{f}} W\right), h=\rho_{0 \mathrm{f}} W\left(c_{0 \mathrm{f}}\right.$ $\left.+s_{\mathrm{f}} W\right)$, and $\rho_{0 \mathrm{t}}$ is the initial density of the target, $\rho_{0 \mathrm{f}}$ is the initial density of the flyer, $c_{0 \mathrm{f}}$ and $s_{\mathrm{f}}$ are the Hugoniot parameters of the flyer. The initial density of the polyimide sample is $\rho_{0 \mathrm{t}}=1.35 \mathrm{~g} / \mathrm{cm}^{3}$, and parameters for flyers are obtained from ref. [1].

The data $\left(D_{i}, u_{i}\right),\left(p_{i}, u_{i}\right)$ of polyimide was measured from our experiments and shown in Table 1. Finally, as shown in Figure 3 , the relationship between $D$ and $u$, which is the Hugoniot curve, is linear fitted with the least square method:

$$
D=c_{0}+s u=(2.62 \pm 0.22)+(1.25 \pm 0.063) u .
$$

So the parameters $c_{0}$ and $s$ for polyimide are determined as $c_{0}=(2.62 \pm 0.22) \mathrm{km} / \mathrm{s}$ and $s=1.25 \pm 0.063$.

The relationship between $p$ and $u$, as shown in Figure 4, is parabola fitted with the least square method:

$$
p=(3.27 \pm 0.44) u+(1.76 \pm 0.12) u^{2} .
$$

For most materials, the Grüneisen parameter can be calculated by the Dugdale-MacDonald model

$$
\Gamma=-\frac{1}{3}-\frac{v}{2} \frac{\mathrm{d}^{2}\left(p_{\mathrm{c}} v^{2 / 3}\right) / \mathrm{d} v^{2}}{\mathrm{~d}\left(p_{\mathrm{c}} v^{2 / 3}\right) / \mathrm{d} v},
$$

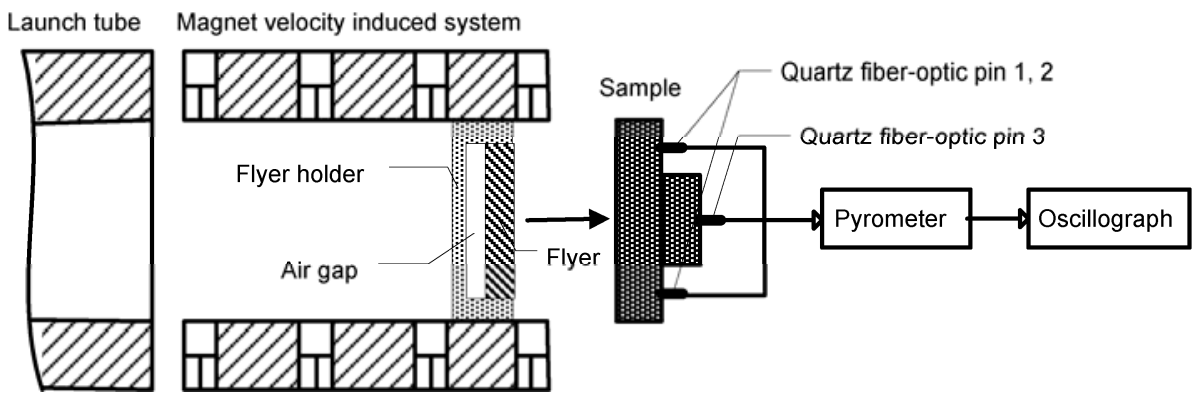

Figure 1 Schematic of experiment system. 


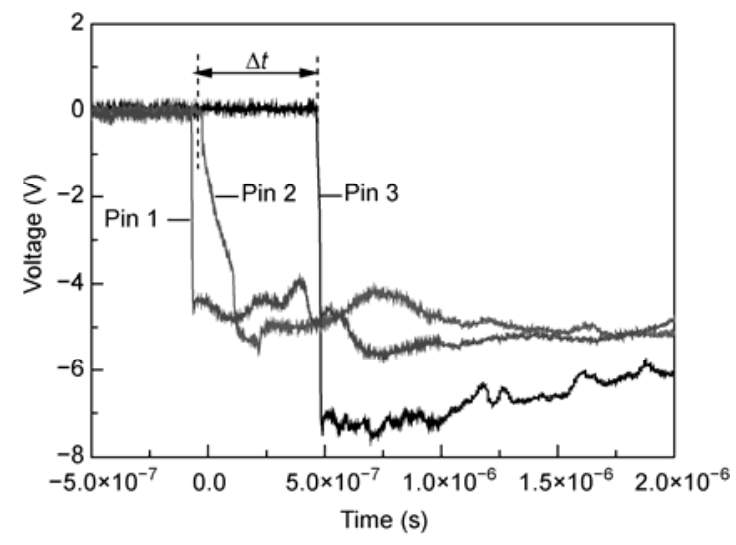

Figure 2 Original signals of the quartz fiber-optic pins.

Table 1 Two-stage light-gas gun experimental data of polyimide

\begin{tabular}{cccccc}
\hline No. & Flyer & $W(\mathrm{~km} / \mathrm{s})$ & $D(\mathrm{~km} / \mathrm{s})$ & $u(\mathrm{~km} / \mathrm{s})$ & $P(\mathrm{GPa})$ \\
\hline 1 & $\mathrm{Al}$ & 1.99 & 4.53 & 1.46 & 8.92 \\
2 & $\mathrm{Cu}$ & 3.30 & 5.89 & 2.78 & 22.1 \\
3 & $\mathrm{Cu}$ & 4.63 & 7.48 & 3.80 & 38.4 \\
4 & $\mathrm{Ta}$ & 4.65 & 7.68 & 4.04 & 41.9 \\
5 & $\mathrm{Ta}$ & 4.74 & 7.75 & 4.12 & 43.1 \\
\hline
\end{tabular}

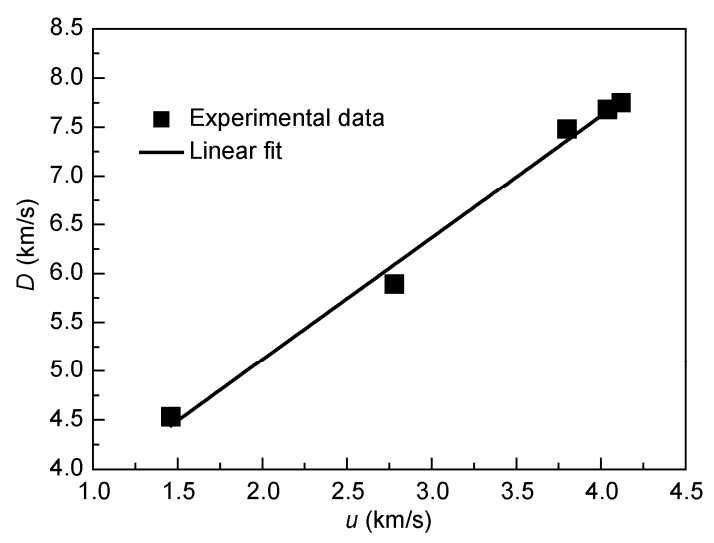

Figure 3 Shock velocity $D$-particle velocity $u$ data for polyimide.

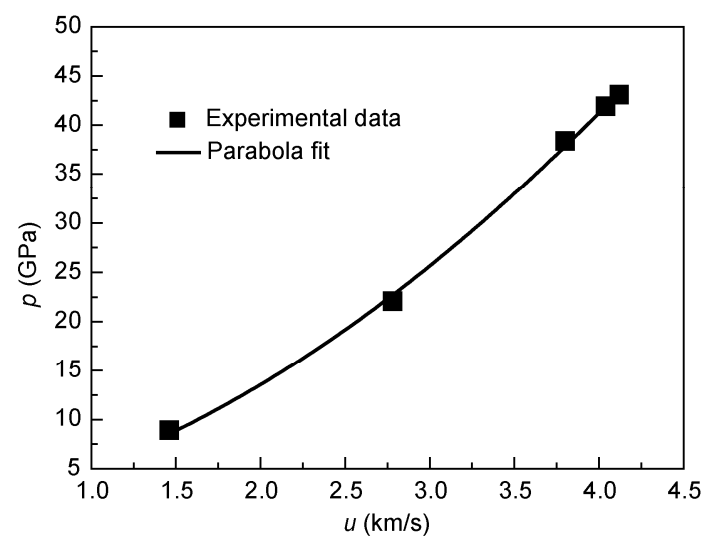

Figure 4 Shock pressure $p$-particle velocity $u$ data for polyimide. where $p_{\mathrm{c}}$ is the cold pressure. At initial state, the eq. (9) can be taken the approximation as $[1,14]$

$$
\Gamma_{0}=2 s-1 \text {. }
$$

As the linear Hugoniot slope coefficient $s=1.25 \pm 0.063$, then the Grüneisen parameter at initial state for polyimide $\Gamma_{0}=1.50 \pm 0.126$.

Huang [15] has given the direct relationship between the Grüneisen parameter and the shock wave parameters as

$$
\Gamma(v)=\frac{D\left(2 D-c_{0}\right)\left[c_{0}+(s-1) D\right]-D c_{0}\left(D-c_{0}\right) / 3-s c_{0}^{3}}{D\left(D-c_{0}\right)\left(D+c_{0}\right)} .
$$

According to the shock wave equations, the relationship among $v, D$ and $u$ is

$$
\frac{v}{v_{0}}=1-\frac{u}{D},
$$

where $v=1 / \rho$ is the specific volume, and $v_{0}=1 / \rho_{0}$ is the specific volume at the initial state.

With the data in Table 1, the values of $\Gamma$ and the corresponding $v / v_{0}$ are respectively calculated by eqs. (11) and (12), and the results are shown in Table 2. Finally, as shown in Figure 5, the relationship between $\Gamma$ and $v_{0} / v$ was fitted by the least square method as

$$
\Gamma=(2.04 \pm 0.089)-(0.51 \pm 0.046) v_{0} / v .
$$

When $v=v_{0}$ in eq. (13), the Grüneisen parameter at the initial state is obtained as $\Gamma_{0}=1.53 \pm 0.135$.

The values of the Grüneisen parameter at initial state calculated by eqs. (10) and (13) are very close, which shows

Table 2 Values of the Grüneisen parameter calculated by experiment data and Huang equation

\begin{tabular}{cccccc}
\hline No. & 1 & 2 & 3 & 4 & 5 \\
\hline$v_{0} / v$ & 1.48 & 1.89 & 2.03 & 2.11 & 2.13 \\
$\Gamma$ & 1.28 & 1.11 & 0.98 & 0.97 & 0.96 \\
\hline
\end{tabular}

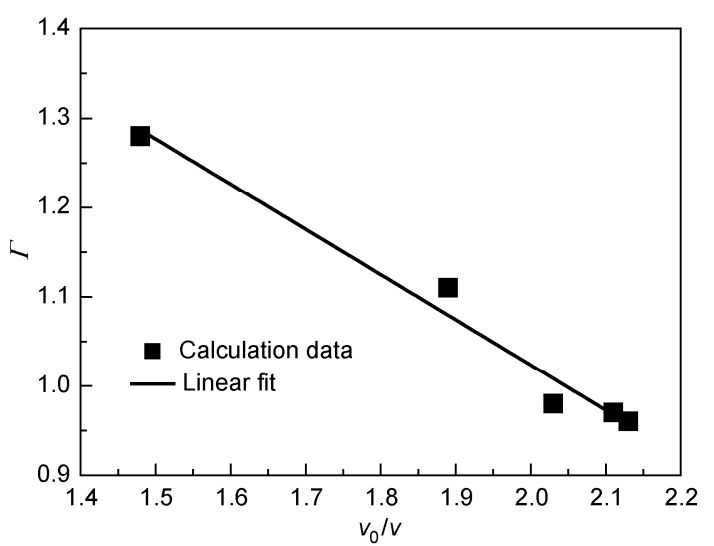

Figure 5 Fitting relationship between the Grüneisen parameter and $v_{0} / v$. 
the reliability of our experiments.

In conclusion, the Mie-Grüneisen EOS based on the shock adiabat for polyimide has been determined with twostage light-gas gun experiment in our research. The Hugoniot curve for polyimide is fitted with the experimental data as $D=c_{0}+s u=(2.62 \pm 0.22)+(1.25 \pm 0.063) u$, and the Grüneisen parameter at initial state is determined as $1.53 \pm 0.135$. The values are proved reliable via the comparison of Grüneisen parameter value calculated from two different theoretical models using the experimental data.

This work was supported by the National Natural Science Foundation of China (11002162 and 10672180). The authors wish to thank the foreign teacher Ranald Macdonald in National University of Defense Technology for his advice on the English writing skills.

1 Tang W H, Zhang R Q. Introduction to Theory and Computation of Equation of State (in Chinese). 2nd ed. Beijing: Higher Education Press, 2008
2 Wang L D, Li Y, Chang C, et al. Chin Sci Bull, 2005, 50: 505-508

3 Thierry P, Bernard D, Denis P. J Spacecr Rockets, 2011, 48: 226-234

4 Roberts C C, Letts S A, Saculla M, et al. Polyimide films from vapor deposition: Toward high strength, NIF capsules. LLNL Technical Report UCRL-JC-132194, 1998

5 Tsai F Y. J Phys D, 2001, 34: 3011

6 Gerald N. Space hypervelocity microparticle impact damage in polymer matrix composites. Dissertation for the Master Degree. Toronto: Toronto University, 1993. 1-15

7 Xiao F, Wang K, Zhan M S, et al. J Mater Sci, 2012, 47: 4904-4913

8 Huang Y X, Tian X B, Lü S X, et al. Appl Surf Sci, 2012, 258: 5810 5814

9 Perinati E, Lotti S, Colasanti L, et al. J Low Temp Phys, 2012, 16: 232-235

10 Shimamura H, Nakamura T. Polym Degrad Stabil, 2010, 95: 21-33

11 Minton T K, Wright M E, Tomczak S J, et al. ACS Appl Mater Interfaces, 2012, 4: 492-502

12 Yokoo M, Kawai N, Nakamura K G, et al. Int J Impact Eng, 2008, 35: 1878

13 Wang R B, Tian J H, He L F, et al. Explosion and Shock Waves, 2006, 26: 284-287

14 Rice M H, McQueen R G, Walsh J M. Solid State Phys, 1958, 6: $1-63$

15 Huang Y K. J Chem Phys, 1969, 51: 2573

Open Access This article is distributed under the terms of the Creative Commons Attribution License which permits any use, distribution, and reproduction in any medium, provided the original author(s) and source are credited. 